\title{
Linear Dark Field Control: Simulation for Implementation and Testing on the UA Wavefront Control Testbed
}

\author{
Kelsey Miller ${ }^{a, b}$, Olivier Guyon ${ }^{a, c, d}$

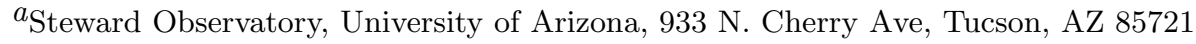 \\ ${ }^{b}$ College of Optical Sciences, University of Arizona, 1630 E. University Blvd., Tucson, AZ 85721 \\ ${ }^{c}$ Subaru Telescope, National Observatory of Japan, National Institutes of Natural Sciences, 650 N. A'ohoku Place, Hilo, \\ HI 96720 (USA)

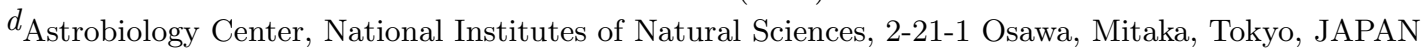

Keywords: wavefront control, speckle nulling, electric field conjugation (EFC), linear dark field control (LDFC)

\begin{abstract}
This paper presents the early-stage simulation results of linear dark field control (LDFC) as a new approach to maintaining a stable dark hole within a stellar post-coronagraphic PSF. In practice, conventional speckle nulling is used to create a dark hole in the PSF, and LDFC is then employed to maintain the dark field by using information from the bright speckle field. The concept exploits the linear response of the bright speckle intensity to wavefront variations in the pupil, and therefore has many advantages over conventional speckle nulling as a method for stabilizing the dark hole. In theory, LDFC is faster, more sensitive, and more robust than using conventional speckle nulling techniques, like electric field conjugation, to maintain the dark hole. In this paper, LDFC theory, linear bright speckle characterization, and first results in simulation are presented as an initial step toward the deployment of LDFC on the UA Wavefront Control testbed in the coming year.
\end{abstract}

\section{INTRODUCTION}

Directly observing exoplanets presents many imaging challenges including high sensitivity and resolution requirements as well as powerful stellar suppression to deal with the large star-planet contrast in the visible and near-IR spectrum. The next generation of ground-based extremely large telescopes (ELTs) and space-based observatories will provide the sensitivity and high resolution required for direct imaging, and multiple coronagraph designs and speckle-nulling techniques will provide high levels of stellar suppression to begin pushing toward the $10^{8}-10^{10}$ contrast levels required by ELTs and space missions respectively for directly imaging Earth-like planets around Sun-type stars in the visible spectrum. To reach such drastic contrast levels, a range of speckle nulling techniques can be used to actively suppress dynamic speckles in the image plane generated by mid-spatial frequencies and build a high contrast dark hole within the science PSF where an orbiting planet could be imaged. ${ }^{3}$ 


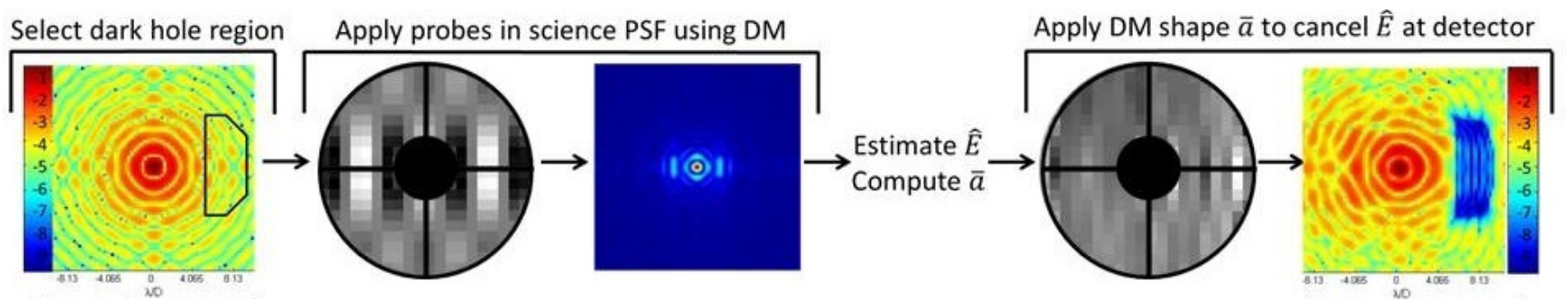

Figure 1: EFC dark hole generation using a Matlab model of the UA Wavefront Control testbed with a Boston Micromachines Kilo DM. The dark hole created here covers a $4 \lambda / \mathrm{D}$ x $10 \lambda / \mathrm{D}$ region centered at $7 \lambda / \mathrm{D}$ from the PSF core and has an average contrast of $10^{-8}$. This method for building a dark hole is described below.

Conventional speckle nulling techniques used for closed-loop speckle control for high contrast imaging require probing to retrieve wavefront information and to sense speckles. EFC is one such technique by which a selected region in the stellar PSF is nulled to high contrast levels, creating a dark hole. To do this, EFC utilizes a system response matrix $G$ containing the differential linear response of the complex focal plane field to wavefront perturbations induced by the DM for a specific set of basis functions; in this work, the basis functions are individual actuator influence functions for all DM actuators located inside the pupil. These actuators are used to build the proper DM shape, $\bar{a}$ that, when multiplied by the system response matrix $G$, cancels the $E$ field at the detector over the selected region in the PSF (see Fig 1). ${ }^{4}$

$$
G \bar{a}+E=0
$$

It is clear from Eq 1 above that, to determine the DM shape $\bar{a}$ necessary to cancel $E$ at the detector over the chosen region in the PSF, the field $E$ at the detector must be known. However, since $E$ is not known, an estimate of the field, $\hat{E}$, must first be derived. To do this, a phase diversity technique is employed. The DM is used to place a minimum of four probes placed across the region in the PSF to be nulled. ${ }^{3}$ For each probe applied, the resulting measured changes in PSF intensity, $\Delta I_{1 \ldots 4}$ coupled with knowledge of the complex probe fields, $R\left(p_{1 \ldots 4}\right)$ and $I\left(p_{1 \ldots 4}\right)$, is used to derive an estimate of the complex field at the detector, $R(\hat{E})$ and $I(\hat{E}){ }^{4}$

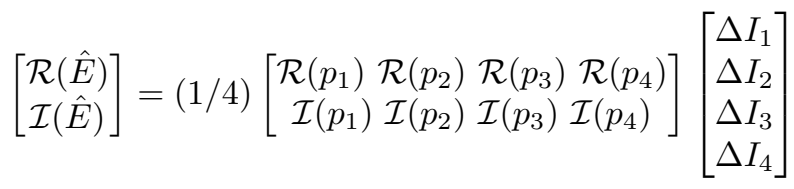

It should be noted that, to reconstruct the phase and amplitude of the complex wavefront $\hat{E}$ and return only real-valued amplitudes for each DM actuator, $\bar{a}$, the response matrix $G$ and the applied probe fields must also be complex. This information is obtained by building both $G$ and the probes in simulation using a model of the full optical system and DM, and separating and storing the resulting complex field at the detector as real and imaginary field components. Once this has been done, and the response matrix $G$ and probe fields $p_{1 \ldots 4}$ have been generated, the changes in intensity $\Delta I_{1 \ldots 4}$ measured at the detector, and an estimate of the complex field $\hat{E}$ has been derived, the shape of the DM $\bar{a}$ needed to cancel the field at the detector $E$ can be determined. Returning to Eq 1, $\bar{a}$ can now be found by taking the pseudoinverse (denoted by ${ }^{+}$) of the complex response matrix $G$ and multiplying it by the complex field estimate $\hat{E}$. The result is a vector of real-valued amplitudes, one for each actuator located in the pupil. ${ }^{4}$ 


$$
\bar{a}=-\left[\begin{array}{l}
\mathcal{R}(G) \\
\mathcal{I}(G)
\end{array}\right]^{+}\left[\begin{array}{l}
\mathcal{R}(\hat{E}) \\
\mathcal{I}(\hat{E})
\end{array}\right]
$$

This process of applying the probes, updating the complex field estimate $\hat{E}$, and recalculating the actuator amplitudes $\bar{a}$ is iterated upon until the desired contrast floor within the chosen region of the PSF is reached. The resulting dark hole, however, is only valid over a finite timescale that is dependent on wavefront variation. Therefore, continuous updates of the complex wavefront estimate $\hat{E}$ at the detector are required in order to rebuild the high contrast dark hole as the contrast degrades. These updates interrupt the signal at the detector, thereby competing with the science measurement. LDFC will eliminate the need for disruptive $\hat{E}$ updates by providing a new way to actively sense and correct for speckles in the dark field that degrade the contrast of the dark hole.

\section{SIMULATION PARAMETERS}

As the initial step in deploying LDFC, this wavefront control technique is first being designed and tested in simulation with the goal of implementing conventional speckle nulling techniques in conjunction with linear dark field control on the University of Arizona Wavefront Control testbed in the near future. To make this transition from simulation to lab bench smoothly, the work presented here was completed using a Matlab simulation of the UA Wavefront Control testbed complete with a deformable mirror and a basic Lyot coronagraph in monochromatic light at the central wavelength of the testbed's white light source, $\lambda=550 \mathrm{~nm} .{ }^{1}$ The centrallyobscured pupil mask on the testbed was used as the entrance pupil for this work, and a model of the testbed's Boston Micromachines Kilo DM served as the simulation's spatial light modulator.

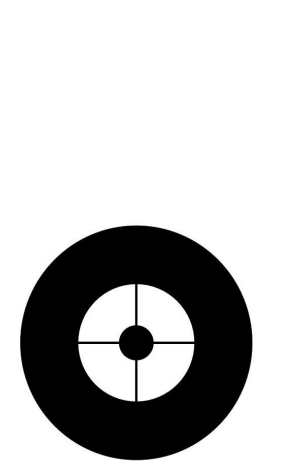

(a) Pupil mask

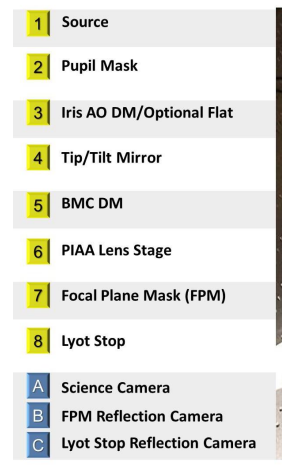

Figure 2: (a) Centrally-obscured pupil used both in simulation and in the lab. (b) Layout of the University of Arizona's Wavefront Control testbed. The Matlab model of this testbed was used for the following work.

\section{ENHANCING HIGH-CONTRAST SPECKLE CONTROL WITH LDFC}

\subsection{LDFC theory}

While conventional speckle nulling techniques like EFC have been proven effective for creating a dark hole, the science interruption caused when updating $\hat{E}$ limits the available, uninterrupted observation time to the period over which the EFC-generated dark hole remains stable at the desired contrast level. By holding bright speckles static, (LDFC) can maintain the dark hole as long as the relationship between the bright and dark speckles remains constant. This relationship will likely hold for long periods of time and will allow LDFC to extend 
the uninterrupted observing time by bypassing the disruptive $\hat{E}$ update process that requires breaking the dark hole. ${ }^{5}$ As a method for stabilizing the dark hole, LDFC is faster, more sensitive, and more robust than using EFC alone. Instead of using probes to build an $E$ field estimate, LDFC continuously re-updates the dark hole by using the EFC-generated dark field as the ideal reference. To update the dark hole as it changes over time, an image is taken at the science detector and the reference image is subtracted off. The updated DM shape $\bar{a}_{\text {update }}$ is calculated using the residual intensity image $\Delta \mathrm{I}$ and the control matrix $C M=R M^{+}$where $R M$ is the response of the bright field intensity at the detector to changes in the pupil.

$$
\begin{gathered}
\Delta I=P S F_{\text {aberrated }}-P S F_{\text {ideal } E F C \text { darkhole }} \\
R M \bar{a}_{\text {update }}+\Delta I=0
\end{gathered}
$$

To ensure a stable control loop, $\Delta I$ must respond linearly to changes in the pupil plane, $A_{\text {pupil }}$, which can be controlled by the deformable mirror. While the image plane complex amplitude is linearly related to the complex amplitude of the pupil plane, the measured intensity $I$ in the image plane, is not. Intensity is the sum of quadratic and cross terms from both the speckle and pupil field contributions in the image plane, $\psi_{\text {pupil }}$ and $\psi_{\text {speckle }}$, and the measured intensity $I$ at the detector is therefore quadratically related to the pupil field contribution in the image plane, $\psi_{\text {pupil }}$.

$$
\begin{gathered}
\psi_{\text {full image field }}=\psi_{\text {pupil }}+\psi_{\text {speckle }} \\
I=\left|\psi_{\text {pupil }}+\psi_{\text {speckle }}\right|^{2}=\left|\psi_{\text {pupil }}\right|^{2}+2 \psi_{\text {pupil }} \psi_{\text {speckle }}+\left|\psi_{\text {speckle }}\right|^{2}
\end{gathered}
$$

However, in the bright field outside of the dark hole, speckles with high amplitude intensity can be approximated to be linearly related to the pupil field contribution in the image plane, $\psi_{\text {pupil }}$, which is the linear transformation, $F$, of the pupil plane complex amplitude, $A_{\text {pupil }}$. The bright speckle intensity $I_{\text {brightfield }}$ can therefore be approximated as linearly dependent on the pupil plane $A_{\text {pupil }}$.

$$
\begin{gathered}
\left|\psi_{\text {speckle }}\right|>\left|\psi_{\text {pupil }}\right| \therefore\left|\psi_{\text {speckle }}\right|^{2}>\left|\psi_{\text {pupil }}\right|^{2} \\
I_{\text {bright field }} \approx 2 \psi_{\text {pupil }} \psi_{\text {speckle }}+\left|\psi_{\text {speckle }}\right|^{2} \\
\psi_{\text {pupil }}=\mathcal{F}\left\{A_{\text {pupil }}\right\} \\
I_{\text {bright field }} \approx 2 \mathcal{F}\left\{A_{\text {pupil }}\right\} \psi_{\text {speckle }}+\left|\psi_{\text {speckle }}\right|^{2}
\end{gathered}
$$

This linear dependence of bright field speckle intensity $I_{\text {brightfield }}$ on the controllable pupil field $A_{\text {pupil }}$ means that sufficiently bright speckles can be used in place of the field estimate $\hat{E}$ to derive continuous pupil field updates without the need for applying disruptive probes in the science image and breaking the dark hole. To use bright field speckles to inform the required pupil plane update applied on the DM, it is first necessary to quantify what intensity amplitude is sufficient to ensure a linear dependence on the controllable pupil field.

\subsection{Characterizing linear bright field speckles}

The first step in implementing LDFC is to determine an intensity threshold above which bright field speckles can be approximated as being linearly related to changes in the pupil field. This limit will inform which regions of the PSF can be reliably used to build a stable closed-loop control system. To do this, the dark hole was first built using EFC and the Matlab model of the UA Wavefront Control testbed with the BMC DM model as the system's spatial light modulator. This unaberrated PSF with its $10^{-8}$ average contrast dark hole was then saved

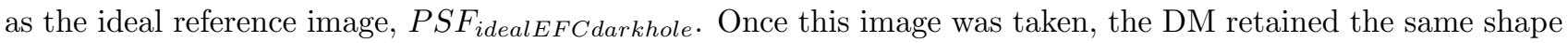
to maintain the dark hole, with the exception of one actuator. The amplitude, $A_{n}$, of this single DM actuator in the pupil was then varied in $n$ incremental steps over a $312 \mathrm{~nm}$ range from $-156 \mathrm{~nm}$ to $156 \mathrm{~nm}$. For each amplitude step, an image of the science PSF, $P S F_{\text {aberrated }}$, was taken and the ideal reference subtracted off, creating $\Delta \mathrm{I}$ (see Eq 4 and Fig 3). 


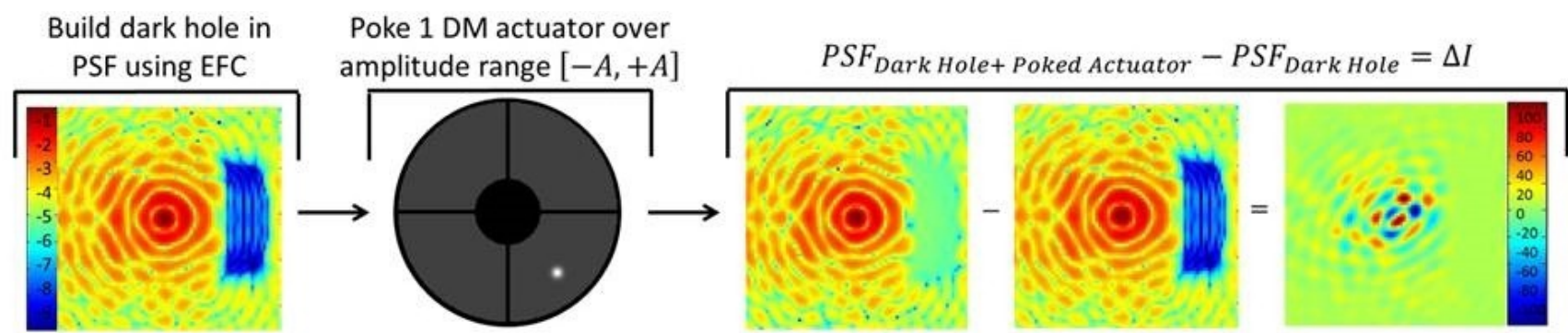

Figure 3: Method by which the response of each pixel to a single actuator amplitude change was determined.

A vector, $\alpha_{\text {pixel }}$, of length $n$ containing $\Delta I / A_{n}$ for each DM amplitude actuation was then built for each pixel in the image. A least-squares fit to a line, $y$, was then computed for each pixel. The fit metric, $\beta$, was set to accept fit pixel response curves values of $0.97 \leq \beta \leq 1.03$ as linear.

$$
\beta=\left(\alpha_{\text {pixel }}^{T} \alpha_{\text {pixel }}\right)^{-1} \alpha_{\text {pixel }}^{T} y
$$

In Fig 4 below, an example response curve is shown for a bright field pixel located between the PSF core and the dark hole. This response curve was calculated over a $312 \mathrm{~nm}$ DM amplitude range and shows where the response begins to go non-linear. The range over which this pixel responds linearly is about $156 \mathrm{~nm}$ between -78 and $+78 \mathrm{~nm}$ in DM stroke amplitude.

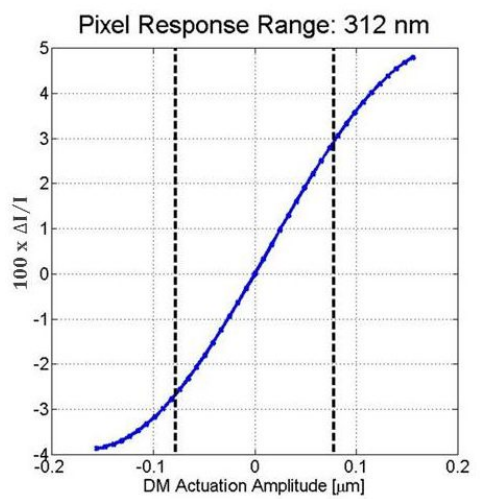

Figure 4: Bright pixel response to linearly increasing DM stroke over $312 \mathrm{~nm}$ range with amplitudes [-156nm, $+156 \mathrm{~nm}]$. The bright field pixel response is linear over a $156 \mathrm{~nm}$ DM stroke range with $\beta=0.998$.

For bright field pixels, this linear response to a $156 \mathrm{~nm}$ stroke range trend was common. Figure 5 depicts response curves for 10 different pixels in the PSF shown in the center image. The $\Delta I$ response of five dark field pixels located within the dark hole to a $156 \mathrm{~nm}$ DM stroke range are plotted in the left plot. Similarly, in the plot to the right, the $\Delta I$ response of five bright field pixels located outside the dark hole are plotted versus the same $156 \mathrm{~nm}$ stroke range. Fitting with theory, pixels within the dark field consistently showed a quadratic relationship to the same pupil change to which bright field pixels showed a linear response over a $156 \mathrm{~nm}$ stroke range on the DM. 


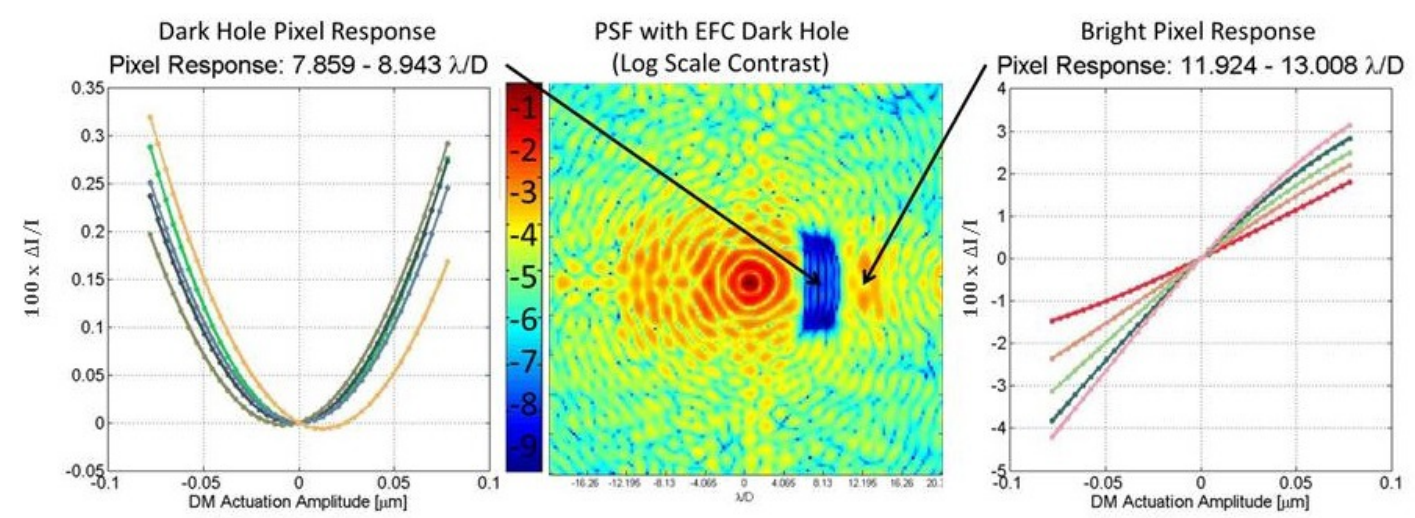

Figure 5: Response curves of dark field pixels versus bright field pixels to the same $156 \mathrm{~nm}$ stroke range pupil change. Clear demonstration of the quadratic dependence of the dark field and the linear dependence of the bright field on amplitude fluctuations in the pupil plane.

From these results, it can be concluded that bright field speckles are, in fact, linearly related to the pupil field and can be used to build a stable closed-loop control system. To utilize this property for building an LDFC control loop, an intensity (contrast) threshold is needed in order to consistently select only speckles with intensities high enough to be linearly dependent on the pupil field. The process of poking the mirror over a $156 \mathrm{~nm}$ range of amplitudes was done repeatedly using single actuators in multiple locations across the mirror. For each run, the linear pixels with the lowest contrast were selected, and the average contrast of these lowest-intensity, linearly-responding pixels was found to be $\sim 10^{-4.8}$. An example of one of these runs is shown in Fig 6 below. A cross section of pixels across the center of the PSF is plotted in green, and all linearly-responding pixels are highlighted in cyan. The dark blue line at the top of the plot is the linear metric $\beta$, on which all linear values of $0.97 \leq \beta \leq 1.03$ are also highlighted in cyan. This plot displays the linear response contrast limit in black.

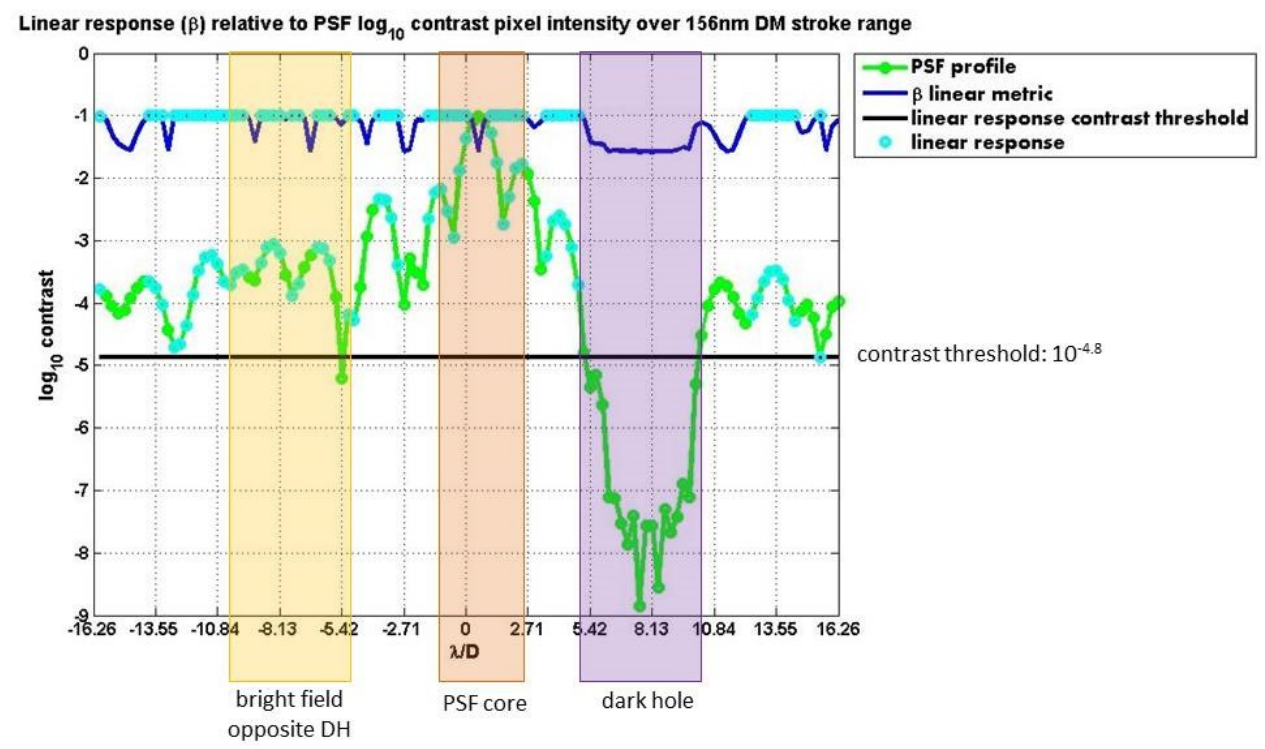

Figure 6: Mapping the linear response of pixels in the bright and dark fields across a central cross section of the PSF in green. Linear metric $\beta$ shown in blue. Linearly responding pixels highlighted in cyan. Minimum contrast level at which pixels still showed a linear response denoted in black at $10^{-4.8}$.

Limiting the pixels used to build the LDFC response matrix $R M$ to only those with a log-scale contrast $>10^{-4.8}$ should ensure that the response matrix is linearly dependent on the pupil field, which should therefore 
also ensure that the LDFC control loop will be linear, stable, and will converge to the ideal contrast floor. This minimum contrast level of $10^{-4.8}$ determined from the pixel response linearity tests was used to build the first iteration of a closed-loop LDFC simulation, the results of which are discussed in the following section.

\section{FIRST RESULTS}

With a minimum contrast limit determined for pixels to respond linearly to changes in the pupil field, a simulation of LDFC was designed in the UA Wavefront Control testbed Matlab simulation. To build the LDFC control matrix $C M$, the dark hole was once again created using the DM, and the PSF was saved as the ideal reference. Within this image, only pixels within the control radius of the DM $(\sim 12 \lambda / \mathrm{D})$ and with a contrast $>10^{-4.8}$ were selected and their positions saved (see Fig 7).

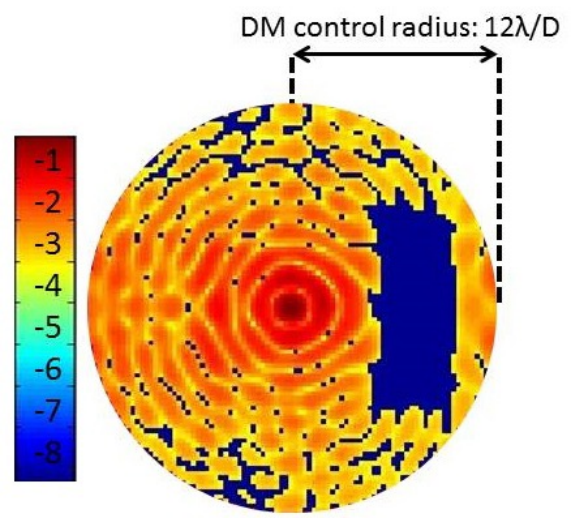

Figure 7: Log-scale contrast map of the bright speckle field pixels used in the LDFC control loop. Pixels selected within the control radius of the DM with a contrast greater than the contrast floor $10^{-4.8}$ set by linearity tests. Pixels not used by LDFC are denoted in dark blue.

A response matrix was then built by poking each actuator on the DM by $1 \mathrm{~nm}$, subtracting off the ideal EFC-created dark hole reference image, and saving the vectorized result in the response matrix $R M$ (see Eq 4). The $R M$ was filtered by only saving the linear bright field pixels in the positions that had been selected from the ideal reference image and discarding the rest. The resulting thresholded $C M_{\text {threshold }}$ was then calculated by taking the pseudoinverse of the filtered $R M_{\text {threshold }}$.

$$
C M_{\text {threshold }}=R M\left(\text { contrast }_{\text {pixel }}>10^{-4.8}\right)^{+}
$$

To test this control matrix, a Kolmogorov phase screen was applied in the pupil, and a wavefront intensity change $\Delta I$ was calculated by taking an image of the resulting aberrated PSF and subtracting off the ideal reference EFC-generated dark hole PSF (see equation 4). The same pixels used to build $C M_{\text {threshold }}$ were selected from the resulting $\Delta I$ image to create $\Delta I_{\text {threshold }}$, which was then multiplied by the filtered command matrix $C M_{\text {threshold }}$ to build the corrected DM shape $\bar{a}_{\text {update }}$.

$$
\begin{aligned}
\Delta I_{\text {threshold }} & =\Delta I\left(\text { contrast }_{\text {pixel }}>10^{-4.8}\right) \\
\bar{a}_{\text {update }} & =-C M_{\text {threshold }} \Delta I_{\text {threshold }}
\end{aligned}
$$

This process was set to continue iterating and updating the DM until the original dark hole average contrast floor of $10^{-8}$ was reached. Results from this first simulation of LDFC demonstrating its ability to stabilize the dark field can be seen below. Figure 8 depicts the applied Kolmogorov phase screens, resulting aberrated PSFs, the LDFC-calculated DM update, and the final resulting dark hole from two separate tests of this technique. 


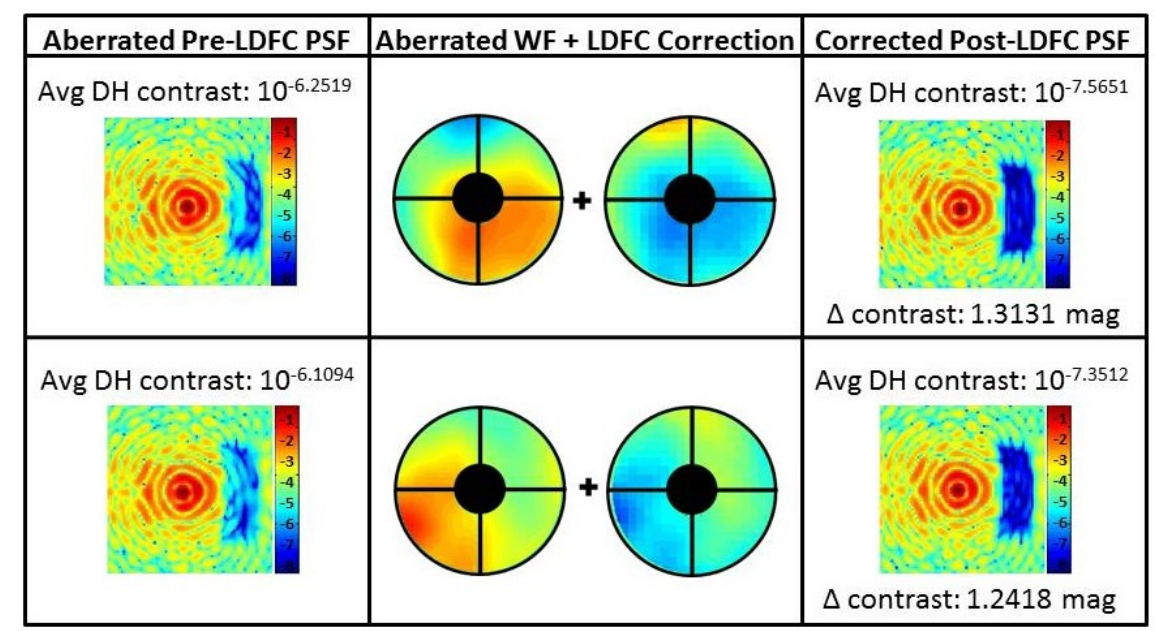

Figure 8: First results showing LDFC's ability to stabilize the dark field by reducing dark field speckle intensity by greater than one magnitude.

These first results show that LDFC is capable of suppressing dark field speckles at $\sim 10^{-6}$ contrast by more than 1 magnitude down to $\sim 10^{-7.5}$. It should be noted that in these tests, the original dark hole average contrast floor of $10^{-8}$ was not quite reached. This is most likely due to a gain factor that needs to be updated with every iteration as the loop closes. It is also possible that the threshold may need to be reset to contrast levels higher than $10^{-4.8}$ and the command matrix rebuilt as the loop converges onto the original contrast floor.

\section{CONCLUSIONS AND FUTURE WORK}

Linear dark field control shows promise as a powerful new method for stabilizing the dark field created with conventional speckle nulling techniques. First results in simulation show that LDFC is capable of suppressing speckles with contrast levels $>10^{-6}$ in the dark field down to the $<10^{-7.5}$ level as seen in Fig 9 below.

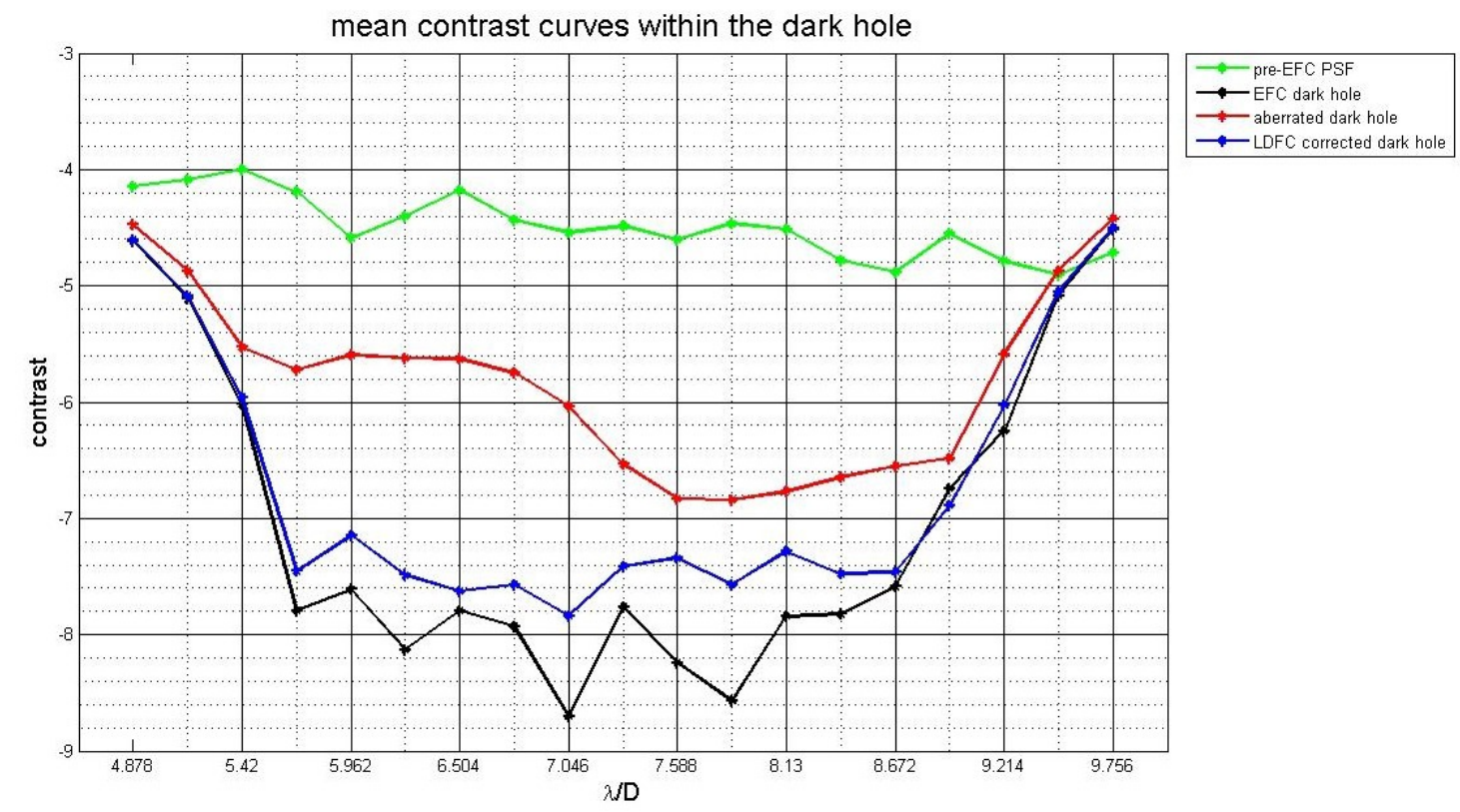

Figure 9: Average dark field contrast across the $\sim 4 \lambda / \mathrm{D}$ wide dark hole for: (1) the intial post-Lyot coronagraph PSF (green), (2) the ideal EFC-generated dark hole (black), (3) the dark hole with speckles induced by a random phase aberration introduced in the pupil (red), and (4) the LDFC-corrected dark hole (blue). 
The next step of this work is to further optimize the control loop to increase the speed of convergence and to reach the contrast floor defined by the ideal dark hole created using conventional speckle nulling methods. Applying higher contrast threshold values to the command matrix as the ideal contrast floor is approached and potentially varying the gain simultaneously should provide the greater convergence speed and deeper contrast levels. Future plans for LDFC also include extension to broadband application and to segmented apertures as well as implementation on the UA Wavefront Control testbed as a first practical lab bench demonstration of LDFC's capabilities.

\section{ACKNOWLEDGMENTS}

Thanks to Sandrine Thomas for her guidance and advice in implementing EFC, to Jared Males for his continued support on this project and editing, and to my fellow lab mates Jennifer Lumbres, Justin Knight and Alexander Rodack for their work on the UA Wavefront Control testbed.

This work is funded by a Technology Demonstration for Exoplanet Missions (TDEM) grant with early support from a NASA Early Stage Innovation grant.

\section{REFERENCES}

1. K. Miller, O. Guyon, J. Codona, J. Knight, and A. Rodack, "Ua wavefront control lab: design overview and implementation of new wavefront sensing techniques," 2015.

2. O. Guyon, "Phase - induced amplitude apodization of telescope pupils for extrasolar terrestrial planet imaging," Astron. Astrophys. 404, p. 379, 2003.

3. T. D. Groff, A. J. Eldorado Riggs, B. Kern, and N. Jeremy Kasdin, "Methods and limitations of focal plane sensing, estimation, and control in high-contrast imaging," Journal of Astronomical Telescopes, Instruments, and Systems 2(1), p. 011009, 2015.

4. A. Give'on, "The electric field conjugation: Controlling amplitude and phase." Jet Propulsion Laboratory, California Institute of Technology, 2009.

5. O. Guyon, J. Codona, K. Miller, J. Knight, and A. Rodack, "Wavefront sensing, control, and psf calibration," February 2015. NASA-JPL LOWFSC Conference. 